CERN-PPE/94-34

23 February 1994

\title{
RADIATION INDUCED EFFECTS ON LEAD AND SCINTILLATING FIBER CALORIMETERS AT THE LHC
}

\author{
G. Anzivino ${ }^{1)}$, J. Bai ${ }^{2,3)}$, C. Bencheikh ${ }^{4)}$, A. Contin ${ }^{5,6,7)}$, R. De Salvo ${ }^{7)}, \mathrm{H} \mathrm{He}^{2,3)}$, \\ L. $\operatorname{Liu}^{2,3)}$, M. Lundin ${ }^{3,8)}$, M.R. Mondardini ${ }^{8)}$, K. Wang ${ }^{3) *}$, X. Xia ${ }^{2,3)}$, C. Yang ${ }^{2,3)}$ \\ and M. Zhao ${ }^{2,3)}$
}

\begin{abstract}
A radiation hardness study of full length (2 meter), lead and scintillating fiber calorimeter test modules is reported. The $0.5 \mathrm{GeV}$ LEP Injector LINAC (LIL) is used as a radiation source. Test modules, containing a number of different fiber types, are subjected to various dose levels and rates in order to observe light loss and annealing effects. Damage assessment shows the importance of irradiation of complete test modules. Loss in fiber light output during irradiation is reported. Irradiated modules are subsequently probed using a CERN PS, $5 \mathrm{GeV}$ electron beam at various times after irradiation. Integrated light attenuation and damage profiles are measured. Performances of a lead/scintillating fiber calorimeter after radiation damage equivalent to four years running at LHC at rapidity two and radius less than 2 meters from the interaction point are predicted.
\end{abstract}

(Submitted to Nuclear Instruments and Methods)

\footnotetext{
1) INFN, Laboratori Nazionali di Frascati, Italy.

2) IHEP, Beijing, People's Republic of China.

3) World Lab Fellowships, Lausanne, Switzerland.

4) Haute Commisariat à la Recherche, Ain-Oussura, Algeria.

5) INFN, Sezione di Bologna, Italy.

6) Università di Bologna, Italy.

7) CERN/LAA, Geneva, Switzerland.

8) Cornell University, Ithaca, NY, USA.

*) Currently at University of California, Irvine, CA, USA.
} 

In developing techniques for the use of lead and scintillating fiber, or "Spaghetti", calorimetry $[1,2,3,4,5,6,7,8]$ in future multi-TeVhadron colliders, the issue of detector radiation "hardness" must be addressed. Examination of radiation-induced stress of the active medium alone, although often useful in the development of improved fiber waveguides and dopants, does not permit the realization of the goal. That is, measurements in circumstances which reflect, as closely as possible, the actual foreseen detector environment are needed. In the following tests, the authors tried to reproduce, as much as possible, the actual experimental conditions of a "Spaghetti" calorimeter in the LHC context. Towards this end:

- Full-size $2 \mathrm{~m}$ long modules, filled with $1 \mathrm{~mm}$ diameter fibers mirrored at one end and with the standard lead-to-fiber filling ratio of $4: 1$, were constructed and tested.

- The radiation particles (electrons) and energy ( $500 \mathrm{MeV}$ ), derived from CERN's Lep Injection Linac (LIL), were chosen such as to reproduce the main source of radiation at LHC, i.e. $\gamma^{\prime}$ s from $\pi^{0}$ decay.

- The total delivered dose $(4 \div 5 \mathrm{Mrad}$ at shower peak) corresponded to the expected dose (for four years of nominal running at an integrated luminosity of $10^{41} \mathrm{~cm}^{-2}$ year $^{-1}$ ) at the geometrical limit (pseudorapidity $=2.5$ and radius less than two meters) of a possible central calorimeter at LHC.

- The longitudinal response of the modules was completely measured before and after irradiation so that the degradation in the performances of the calorimeter (in terms of energy resolution and light output) could be precisely evaluated.

The LIL irradiation source provided up to $10^{10} 0.5 \mathrm{GeV}$ electrons per burst with a repetition rate of up to $100 \mathrm{~Hz}$. The electron range matches the energy range of the expected LHC background and the LIL's flexibility and power allowed irradiation at any required rate. Irradiations were made in a parasitic mode using LIL during the standby periods between LEP fillings.

A bunker was specially constructed to allow the use of the LIL beam as an irradiation source for these measurements. The bunker was located directly on the axis of LIL, just after the bending magnet that steers electrons and positrons to the two injection branches of the LEP Electron Positron Accumulator (EPA). A remote controlled movement allowed the successive presentation of different modules to the beam line. The bunker was equipped with a beam dump to allow LIL beam development.

Four calorimeter modules (each containing four $3.6 \times 3.7 \mathrm{~cm}^{2}$ cells with one type of fiber per cell) were built and filled with fibers loaded with different wavelength shifting dopants and coated with either standard or "Radiation hard" cladding ${ }^{1)}($ see

1) The term "Radiation hard" refers to fibers with fluorinated PolyMethyl-MetaAcrylate (PMMA) cladding while "Standard" refers to fibers with non-fluorinated PMMA cladding. 
Table 1).

The results presented here concern modules 2, 3 and 4. Results on module 1, built and irradiated in 1991, are published elsewhere [9].

The modules' $7.5 \times 8 \mathrm{~cm}^{2}$ front faces were uniformly exposed to the LIL beam with a raster scan where the vertical swing was obtained by steering in 7 columns and the horizontal swing by cycling a remote-controlled movement in 11 lines. In order to avoid shower channeling in the fibers, the calorimeter modules were slightly tilted away from the incoming beam direction.

The light output from each of the four cells was measured during the entire irradiation period using Hamamatsu photodiodes (S1227-1010BR) coupled to the fibers via standard light guides. Only the measurements corresponding to the beam impinging at the center of each cell were used to monitor the damage during irradiation (see section 2). A monitor of the beam intensity, provided by LIL, allowed the measurement of the delivered dose and the burst-by-burst normalization of the light output from the cells.

During irradiation, Argon or compressed air were flown from the back of the modules along the fibers to avoid possible long-range radio-chemical damage effects. Subsequent tests at the PS with $6 \mathrm{GeV}$ electrons shot at various positions at $90^{\circ}$ w.r.t. the fiber longitudinal axis allows for a comparative measurement of the signal degradation due to radiation damage along the fibers. Electrons were selected using two Cherenkov counters and a $2 X_{0}$ preshower detector placed near the module. The lateral beam size $(\leq 2 \mathrm{~cm})$ was defined by a wire chamber. The fibers were read out by standard photomultipliers (Philips XP2008). Both the global response and (for a small sub-sample of events) the signal shapes were recorded in order to separate the direct (light traveling from the beam impact point directly to the phototube readout) and the reflected pulses (light initially traveling in the opposite direction which reflects off of the fibers' mirrored surface and returns to the phototube readout). These measurements will allow one to determine, for each fiber, the relative importance of the two components (attenuation and production) of the radiation damage. These data will be presented in a future publication.

The PS fiber degradation measurements, fed as starting conditions in a Monte Carlo, allow the evaluation of the calorimeter performance degradation due to irradiation. This damage evaluation technique was chosen because it would have been too cumbersome to build and irradiate calorimeters large enough to directly measure the performance degradation. In small calorimeters, the performance changes would be masked by lack of shower containment.

\section{Irradiation at LIL}

The longitudinal profile of the radiation deposition from $500 \mathrm{MeV}$ electrons inside the calorimeter, as computed by Monte Carlo simulations, is shown in Figure 
1. The dose at the peak of the shower was then calculated using the profile function:

$$
\frac{d E}{d z}=\left(\frac{z}{X_{0}}\right)^{1.9+0.54 \log E} \exp \left(-0.49 \frac{z}{X_{0}}\right),
$$

with $X_{0}=0.71 \mathrm{~cm}$ and $E=0.5 \mathrm{GeV}$. The dose discussed here is the average dose on the whole calorimeter. No attempt is made to differentiate the doses in lead and in fibers, because it would be too difficult to take into account both the specific energy energy loss differences and the secondary irradiation from the lead X-ray fluorescence and photoelectric $\beta$ emission to the surface of the fibers. As pointed out by the RD1 group [13], taking into consideration the specific energy loss differences would raise the peak exposure value of the fibers by a factor 1.7 . This effect might be minor when compared with secondary (soft but intense) irradiation from the lead (responsible for a good fraction of the compensation mechanism). This secondary irradiation would introduce a radial dose gradient across the fibers' diameter and a second, hefty, overall dose correction factor. None of these corrections is done because the relevant quantity in designing a calorimeter is the integrated energy deposited per unit surface. In the following figures and text, the peak doses inside the calorimeter are indicated next to the energy deposition per unit surface, simply as a convenience for the reader.

Table 2 gives the energy deposition (and peak dose) rates per unit surface during three irradiation runs (August, September and December 1992). Module 2 was irradiated in the first run, modules 2,3 and 4 in the second and modules 2 and 3 in the third. Figures 2 a to $2 d$ show the normalized signal as a function of energy deposition per unit surface (and dose) for the four cells of module 2, during the first irradiation period.

Several considerations can be made from these and similar data, collected for each fiber and irradiation period.

- Recovery from radiation damage.

We did not carry out systematic studies of the recovery from radiation damage, because we had a limited access to our experimental set-up at LIL. However, as we periodically interrupted the irradiation to allow LEP filling, we were able to draw some conclusion on the recovery in different fibers. Tables 3 and 4 summarize the behavior of the normalized signal from the different fibers after irradiation and after various recovery periods. A fast and strong recovery takes place for all fibers. Moreover, the rate of recovery seems faster for the most resistant fibers. The fact that recovery is observable depends on the very high dose rate delivered in the first two irradiation periods. Fibers irradiated with a much lower dose rate during the third period do not show any progressive loss (see Figure 3, beyond $\left.1.6 \times 10^{12} \mathrm{GeV} / \mathrm{cm}^{2}(0.5 \mathrm{Mrad})\right)$, which indicates that, already at this level of $10^{2}$ times the foreseen LHC maximum rate, a continuous recovery nearly compensates for the damage.

- Comparative study of different fibers. 
From the comparison of the plots on light output as a function of the delivered dose and from the data of Tables 3 and 4 (after recovery) we can conclude that one fiber (3HF 500ppm - radiation-hard cladding) clearly stands out.

\section{Measurements at the CERN PS}

The attenuation curves of modules 2, 3 and 4 before irradiation were measured in November 1991 and in May 1992. Module 2 was measured at the PS about 10 hours after the first irradiation period was completed. Modules 3 and 4 were measured about 60 to 120 hours after the second irradiation period. Typical measurements before and after the damage are shown in Figs. $4 \mathrm{a}$ and $4 \mathrm{~b}^{2)}$.

The irradiation produces two kinds of damage: a loss in light transmission which decreases the apparent attenuation length of the fiber due to the suppression of the reflected component of the signal, and a loss in light production which lowers the response of the fiber in the damaged region. It has already been observed [14] that there are multiple attenuation components in a fiber waveguide. The plots of Figure 4 (and similar plots for all the other fibers) were fit with an attenuation function containing two components: a first exponential to characterize the "short" attenuation component (light which typically traverses less than one meter of fiber between the excitation point and the readout point) and a second exponential to characterize the "long" component (light which traverses a length of fiber greater than one meter). The "long" component is characterized in a way that also takes into account light which traverses the fiber in the direction opposite from the phototube readout. This light is then reflected off of the fibers' mirrors and returns, after travelling a distance of fiber greater than the total length of the fibers, to the readout:

$$
\operatorname{Signal}(z)=p_{1} e^{-z / \lambda_{1}}+p_{2}\left(e^{-z / \lambda_{2}}+R \cdot e^{-(2 L-z) / \lambda_{2}}\right),
$$

where $z$ is the distance from the photomultiplier, $p_{1,2}$ are normalization coefficients, $R$ is the reflection coefficient of the mirror, $L$ is the length of the fiber $(=220 \mathrm{~cm}), \lambda_{1}$ is the attenuation length of the "short" component of the light and $\lambda_{2}$ is the attenuation length of the "long" component of the light. We obtain values of $\lambda_{1}$ between 20 and $40 \mathrm{~cm}$ and values of $\lambda_{2}$ between 1 and $3 \mathrm{~m}$ for the different fibers.

For irradiated fibers, the fit was limited to the range $z<180 \mathrm{~cm}$. The ratio between the data points and the extrapolated fit in the damaged region $(z>180 \mathrm{~cm})$ is shown in Figure 5.

The theoretical damage profile given in (1), also shown in Figure 5, largely differs from the effective damage profile, as already observed in previously reported results [9] and confirmed by careful examination of the data shown elsewhere [13]

2) Note that, while data was collected for all fibers listed in Table 1, the authors present only one of the best and one of the worst of these in graphic form. Results from others are summarized in table format. 
in similar experimental conditions. In general, one would expect that the shape of damage follows the shape of dose delivered. This difference is addressed in Section 4.

In order to evaluate the effect of the damage on the performances of a calorimeter, we parameterized the data in Figure 5 with the function:

$$
\frac{\operatorname{Signal}(z)}{\operatorname{Fit}_{z<180}(z)}=1-p_{1} e^{-(L-z) / p_{2}}+p_{3}\left(e^{-(L-z) / p_{4}}\right)^{2},
$$

where $p_{1, \ldots, 4}$ are free parameters, which better reproduces the effective damage profile. Equations (2) and (3) were used to introduce radiation damage effects into a Monte Carlo program, based on the GEANT package, which describes our lead/scintillating fiber calorimeter and that proved to precisely reproduce the measured performance of our larger prototypes. The main results of these Monte Carlo simulations are the estimation of radiation-damage-induced constant terms to be added in quadrature to the electromagnetic energy resolution and of the reductions in light output. Table 5 shows these two quantities for the different fibers and for $50 \mathrm{GeV}$ incident electron energy.

Figure 6 shows the added term in the energy resolution due to the damage as a function of the incident electron energy for two fibers: $3 \mathrm{HF} 500 \mathrm{ppm}$ fibers with radiation hard cladding and SCSF81Y9 fibers with standard cladding. Some comments are in order.

The added constant term in the energy resolution is due to the profile of the damage in light production. The steeper the damage is as a function of depth, the larger is the added resolution term because the fluctuations in the starting point of the shower become more important. As a consequence, the added term increases at low energy, where the shorter showers develop within the highly damaged region. The reduction in light output due to the damage in light transmission is nearly constant as a function of energy, since it depends mainly on the quantity of reflected light which survives the damaged region. On the contrary, the damage in light production induces a non-linearity in the response of the calorimeter as a function of energy which also depends on the intensity and on the profile of the damage. All fibers present light output losses; the percent of surviving light yield after irradiation is shown in Figure 7 as a function of energy.

Six months (deemed to be suitable for the observation of long term annealing effects) after all modules underwent their various irradiations they were again taken to the CERN PS and their longitudinal scan characteristics were again measured. In addition, the phototube gain of some sub-modules was controlled in such a way that allowed normalization of total light output for comparisons. This was achieved in the following manner. Damage in each module was characterized with a longitudinal scan (as shown in Figure 4b). The scans were done with phototube readouts set at arbitrary values with respect to one another, this to allow full use of the ADC's dynamic range. For gain cross calibration, one point along the modules' longitudinal axis was then 
selected and the various fiber types' signals were readout with the same phototube, set at a fixed gain. The point chosen $(90 \mathrm{~cm}$ from the phototube readout) was well outside of any primary radiation damage effects. The primary source of variability of results in this measurement was observed to be differences in light collection efficiency, caused by replacement of the phototube and the light guide. Repetition of this measurement procedure showed its reproducibility to be within $10 \%$ RMS.

The fibers' longitudinal response was characterized by equation (2) in the region closer than $180 \mathrm{~cm}$ to the phototube readout (or more than $40 \mathrm{~cm}$ from the mirrored end of the fibers) and by a combination of equations (2) and (3) in the remaining region near the fibers' mirrored ends. The fits were then normalized at $90 \mathrm{~cm}$ using the cross calibration point measured.

Figure 8 shows the longitudinal scans of two such fibers $(500 \mathrm{ppm} 3 \mathrm{HF}$ with fluorinated cladding represented by clear triangles and SCSF38 with standard cladding represented by darkened circles) after normalization. The fits, also after normalization, are overlaid. Both measurements were made without the use of a yellow cut-off filter.

This normalization makes it possible to compare the light output (in arbitrary units) between various fibers and at various distances from the damaged region. Table 6 summarizes four green 3HF fibers and one blue fiber (SCSF38) at three positions; at the phototube readout (far from the radiation damaged region), at the normalization point $(90 \mathrm{~cm}$ from the phototube readout end of the fibers) and at the mirrored surface of the fibers (where damage primarily begins to occur).

The $3 H F$ fibers received a total peak dose of 9.7 Mrads, while the SCSF 38 fiber received only 4.9 Mrads. The difference between the two types is apparent. SCSF38 provides a greater light output than any of the $3 H F$ fibers. However, this fiber is generally characterized by large attenuation effects which are greatly enhanced by radiation damage. The use of a yellow cut-off filter would improve the attenuation length (by eliminating the highly attenuated, low wavelength portion of the emission spectrum) of the blue fiber, but at the price of a reduction in total light yield.

\section{Discrepancy Between Dose Deposition Profiles and Damage Profiles}

As it was stated in Section 3 and shown graphically in Figure 5, a significant difference between the dose profile and the damage profiles measured in longitudinal scans has been observed. This difference does not affect previously shown calculations of degradation in calorimeter light production and electromagnetic energy resolution due to irradiation, since the attenuation curves, inclusive of measured damage profiles, were used in the Monte Carlo and were thus independent from interpretation.

Comparison of the radiation induced loss in total light output, measured at a given point along the fibers' longitudinal axis (information that can be taken, for instance, from Figure 5), to the dose delivered to that same point inside of the radiation damage module (information derived from the data shown in Figure 1, with longitu- 
dinal depth greater than that at local dose peak), reveals several interesting pieces of information. Figure 9 shows the observed percent loss in light output as a function of local integrated dose for two fibers; SCSF81Y9 and $500 \mathrm{ppm} 3 \mathrm{HF}$ with radiation hard, fluorinated cladding. Data was taken from fibers irradiated to a peak of 4.8 Mrads (500 ppm 3HF, darkened circles) and 4.1 Mrads (SCSF81Y9, clear circles) and measured 10 hours and 60 hours (respectively) afterwards.

Several clear effects are observed:

- A major fraction of the damage seen by the fibers occurs in the first few tenths of a Mrad. SCSF 81 Y9 is observed to have undergone a loss in total light output of roughly $35 \%$ at the point of the fibers which received 4 Mrads. However, one half of that light output loss was observed to have occurred in the first two tenths of a Mrad. Similar results are observed when considering the $3 \mathrm{HF}$ fiber with radiation hard cladding.

- It is also noted that the damage observed has a non-linear relationship with the delivered dose. Rather, the same data plotted on a log-log scale in Figure 10 shows that damage goes as a power function of the dose.

Notice that this saturation effect could not be the result of damage delivered directly to the fibers' mirrors. Any degradation of the mirrors, seen as a percent loss in the mirrors reflectivity, is a constant fraction throughout the measurement.

The reflected component of light must also twice traverse the damage region in its journey to the phototube readout. Color centers, which occur as a function of irradiation delivered to a given point in the damaged region would act as a neutral density filter whose light reduction ability would depend on the distance and extent of damage through which the light travels. In effect this loss in light transmittance of the fiber is not a constant fraction as a function of where the fiber excitation occurred and would serve to hide the saturation effect rather than exaggerate it. Thus, the only significant issue involved in considering fiber damage saturation due to irradiation is light production or emission.

It should also be stated here that these results do not explain a previously observed [9], secondary radiation damage effect whose characteristic was to extend damage far out of the region in which the irradiation source could have deposited energy. This due to the fact that the scales of length are different by one order of magnitude in the two cases. In addition, as was previously mentioned, during the irradiations reported here, compressed air or Argon was flown from the back of the modules towards the damaged area in order to prevent possible secondary, radiochemical effects. No secondary effects are thus far apparent in irradiations done under these conditions.

\section{Conclusions}

The conclusion from the data presented in Section 3 is that a calorimeter filled with the $3 \mathrm{HF}$-based fibers can withstand, without a significant degradation in per- 
formances, the very high radiation level expected in the barrel calorimeter of a future LHC experiment $\left(3 \div 10 \times 10^{12} \mathrm{GeV} / \mathrm{cm}^{2} /\right.$ year $\sim 1 \div 3 \mathrm{Mrad} /$ year $)$ at an integrated luminosity of $10^{41} \mathrm{~cm}^{-2}$ year ${ }^{-1}$ and at $\left.\eta \sim 2.5\right)$. Results presented here on the electromagnetic energy resolution do not consider possible contributions from photonstatistics. The high light production of the fibers we presently use (about 320 [15] photoelectrons $/ \mathrm{GeV}$ with SCSF38 equipped with yellow Kodak Wratten \#3 filters or SCSF81 without filters, more for 3HF fibers; all of which are far from the photonstatistics limit) should prevent such problems.

It must also be taken into account that the recovery effect, continuously present during irradiation, which can improve significantly the figures presented here, is still not well understood. Irradiations at very low dose rate are therefore of great importance and constitute the main part of our future irradiation program.

Finally, observations have been made regarding the discrepancy between dose profiles and damage profiles in test modules built using foreseen construction techniques. The noted saturation of damage as a function of dose will, in future, be studied at various dose rates.

\section{Acknowledgements}

The authors are indebted to L. Rinolfi of the CERN PS Division for his invaluable help in adapting LIL to our needs. In addition, we would like to thank J.P. Poitier, also from the CERN PS Division, for his stimulating suggestions and support. In general, we would also extend thanks to the entire LIL staff for so kindly accepting us inside their machine. The RD25 group is also indebted to the mechanical expertise of D. Brozzi of the CERN PPE Division.

This study was performed by the RD25 group at CERN, working in the framework of the LAA project.

\section{References}

[1] R. DeSalvo et al., Nucl Instr. and Meth. A279 (1989) 467.

[2] D. Acosta et al., Nucl Instr. and Meth. A294 (1990) 193.

[3] D. Acosta et al., Nucl Instr. and Meth. A302 (1991) 36.

[4] D. Acosta et al., Nucl Instr. and Meth. A305 (1991) 55.

[5] D. Acosta et al., Nucl Instr. and Meth. A308 (1991) 481.

[6] B. Bencheikh et al., Nucl Instr. and Meth. A315 (1992) 354.

[7] B. Bencheikh et al., Nucl Instr. and Meth. A323 (1992) 398.

[8] G. Anzivino et al., Results on a Fully Projective Lead/ Scintillating Fibers Calorimeter, presented at the IV International Conference on Calorimetry in High Energy Physics, Isola d'Elba, Italy, 19-25 September 1993.

[9] G. Anzivino et al., Radiat. Phys. Chem. 41 (1992) 283.

[10] KURARAY Int. Corp., 200 Park Avenue, New York, NY 10166, USA.

[11] BICRON, P.O. Box 271, 2410 AG Bodegraven, The Netherlands. 
[12] G. Anzivino et al., RD25 Collaboration Status Report, CERN/DRDC/93-26, 5 May 1993.

[13] The RD1 Collaboration Status Report, CERN/DRDC 93-16, 10 March 1993.

[14] N.A. Amos et al., Nucl. Instr. and Meth. A297 (1990) 396-403.

[15] B. Bencheikh et al., Nucl Instr. and Meth. A315 (1992) 349. 


\section{Figure Captions}

1. Monte Carlo generated longitudinal dose profile inside our calorimeters. The line is the profile function of equation (1).

2. Normalized fiber response to irradiation bursts as a function of the accumulated energy deposition per unit surface for the August 1992 irradiation of module 2. a) $3 H F$ F $500 \mathrm{ppm}$ with radiation hard cladding; b) $3 H \mathrm{~F} 500 \mathrm{ppm}$ with standard cladding; c) $3 \mathrm{HF} 200 \mathrm{ppm}$ with standard cladding; d) $3 \mathrm{HF} 1000 \mathrm{ppm}$ with standard cladding. The rise in response at $8.6 \times 10^{12} \mathrm{GeV} / \mathrm{cm}^{2}(2.7 \mathrm{Mrad}$ peak $)$ is caused by annealing which occurred during a five hour gap in irradiation.

3. Normalized fiber response to irradiation bursts as a function of the accumulated energy deposition per unit surface for the December 1992 irradiation of module 2. a) $3 H F 500 \mathrm{ppm}$ with radiation hard cladding; b) $3 \mathrm{HF} 500 \mathrm{ppm}$ with standard cladding; c) $3 H F$ 200ppm with standard cladding; d) $3 H F$ F $1000 \mathrm{ppm}$ with standard cladding. Up to $1.6 \times 10^{12} \mathrm{GeV} / \mathrm{cm}^{2}(0.5 \mathrm{Mrad})$, the irradiation rate was $5.1 \times 10^{11} \mathrm{GeV} / \mathrm{cm}^{2} /$ hour $(159 \mathrm{krad} /$ hour $)$. After a 2 hour gap, which causes a recovery in the fiber output, the irradiation was continued with a factor 10 lower rate. The last two points in each plot corresponds to a measurement done after 70 hours from the end of the low dose rate irradiation.

4. Measurement at the PS of the response along the fiber (top) before and (bottom) after irradiation, for $3 H F$ F00ppm fibers with radiation hard cladding (grey circles) and for SCSF81Y9 fibers with standard cladding (clear circles). The irradiation was of $15.4 \times 10^{12} \mathrm{GeV} / \mathrm{cm}^{2}(4.8 \mathrm{Mrad})$ for the $3 \mathrm{HF}$ fibers, and $13.1 \times 10^{12} \mathrm{GeV} / \mathrm{cm}^{2}(4.1 \mathrm{Mrad})$ for the SCSF $81 Y 9$ fibers.

5. Ratio between the data points of Figure $4 \mathrm{~b}$ and the extrapolated fit in the damage region $(z>180 \mathrm{~cm})$, showing the effect of the damage in light production, for $3 \mathrm{HF}$ $500 \mathrm{ppm}$ fibers with radiation hard cladding (top, grey circles) and for SCSF81Y9 fibers with standard cladding (bottom, clear circles). The dashed line is a fit to the data, the dot-dashed line is the energy deposition profile which is overlaid to illustrate the difference between the two shapes.

6. Monte Carlo results on the additional constant term for $3 \mathrm{HF} 500 \mathrm{ppm}$ fibers with radiation hard cladding (darkened circles) and for SCSF81Y9 fibers with standard cladding (clear circles), as a function of the incident electron energy.

7. Monte Carlo results on the ratio between the signals before and after irradiation as a function of the incident electron energy for two fibers: a) darkened circles, $3 \mathrm{HF} 500 \mathrm{ppm}$ fibers with radiation hard cladding; b) clear circles, SCSF 81 Y9 fibers with standard cladding.

8. Two fibers normalized to each other at $90 \mathrm{~cm}$ from the phototubes. Triangles represent $500 \mathrm{ppm} 3 \mathrm{HF}$ with radiation hard cladding; darkened circles represent SCSF 38 with standard cladding.

9. Damage received as a function of dose delivered for two fibers; SCSF81Y9 (clear 
circles) and $500 \mathrm{ppm} 3 \mathrm{HF}$ with fluorinated, radiation hard cladding (darkened circles).

10. Damage received as a function of dose delivered for two fibers; SCSF81Y9 (clear circles) and $500 \mathrm{ppm} 3 \mathrm{HF}$ with fluorinated, radiation hard cladding (darkened circles). Same data as in Figure 9 but in a log-log format. 


\section{Table Captions}

1. Radiation damage test modules - fiber filling.

2. Rates of energy deposition per unit surface and of peak dose in the calorimeter for the different irradiation runs.

3. Signal before and after recovery for module 2, presented as a percentage of the signal observed at the beginning of irradiation.

4. Signal before and after recovery for modules 3 and 4, presented as a percentage of the signal observed at the beginning of irradiation.

5. Reduction in light output and added constant term in the energy resolution (for $50 \mathrm{GeV}$ incident electrons) due to the radiation damage of the fibers, after irradiation to $15.4 \times 10^{12} \mathrm{GeV} / \mathrm{cm}^{2}(4.8 \mathrm{Mrad})$ for the $3 \mathrm{HF}$ fibers and to $13.1 \times$ $10^{12} \mathrm{GeV} / \mathrm{cm}^{2}(4.1 \mathrm{Mrad})$ for the others.

6. Response of four green and one blue fiber normalized at the point $90 \mathrm{~cm}$ from the phototube readout. Notice that the units are arbitrary with the $90 \mathrm{~cm}$ point of SCSF 38 taken to be 100 . 


\begin{tabular}{|l|l|l|}
\hline \hline \multicolumn{2}{|c|}{ Table 1 } \\
\hline Module 1 & SCSF38 PMMA cladding ("Standard") & {$[10]$} \\
& SCSF38 Fluorinated PMMA cladding ("Rad. hard") & {$[10]$} \\
& SCSF81 PMMA cladding ("Standard") & {$[10]$} \\
& SCSF81 Fluorinated PMMA cladding ("Rad. hard") & {$[10]$} \\
\hline Module 2 & 3HF 200 ppm PMMA cladding ("Standard") & {$[10]$} \\
& 3HF 500 ppm PMMA cladding ("Standard") & {$[10]$} \\
& 3HF 500 ppm Fluorinated PMMA cladding ("Rad. hard") & {$[10]$} \\
& 3HF 1000 ppm PMMA cladding ("Standard") & {$[10]$} \\
\hline Module 3 & SCSF38 PMMA cladding ("Standard") & {$[10]$} \\
& SCSF81 Y7 PMMA cladding ("Standard") & {$[10]$} \\
& SCSF81 Y8 PMMA cladding ("Standard") & {$[10]$} \\
& SCSF81 Y9 PMMA cladding ("Standard") & {$[10]$} \\
\hline Module 4 & SCSF38 PMMA cladding ("Standard") & {$[10]$} \\
& SCSF38 PMMA cladding ("Standard") & {$[10]$} \\
& SCSF38 PMMA cladding ("Standard") & {$[11]$} \\
\hline
\end{tabular}

\begin{tabular}{|l|c|c|c|}
\hline \hline \multicolumn{3}{|c|}{ Table 2 } \\
\hline Period & $\begin{array}{c}\text { Hours from start } \\
\text { of irradiation }\end{array}$ & $\begin{array}{c}\text { Energy deposition rate } \\
\left(\frac{\mathrm{GeV}}{\mathrm{cm}^{2}} / \text { hour }\right)\end{array}$ & $\begin{array}{c}\text { Dose rate } \\
(\mathrm{kr} \text { ad } / \text { hour })\end{array}$ \\
\hline August 1992 & $0.7 \div 5.3$ & $19 \times 10^{11}$ & 585 \\
& $9.8 \div 13.9$ & $17 \times 10^{11}$ & 517 \\
\hline September 1992 & $0 \div 18.5$ & $6.1 \times 10^{11}$ & 190 \\
& $33.3 \div 37.0$ & $3.8 \times 10^{11}$ & 120 \\
& $83.0 \div 86.1$ & $0.76 \times 10^{11}$ & 23.7 \\
\hline December 1992 & $0 \div 2.9$ & $5.1 \times 10^{11}$ & 159 \\
& $4.9 \div 26.2$ & $0.46 \times 10^{11}$ & 13.3 \\
\hline
\end{tabular}




\begin{tabular}{|c|c|c|c|}
\hline \multicolumn{4}{|c|}{ Table 3} \\
\hline Fiber & $\begin{array}{c}\text { Relative } \\
\text { signal after } \\
8.6 \times 10^{12} \frac{\mathrm{GeV}}{\mathrm{cm}^{2}} \\
(2.7 \text { Mrad })(\%)\end{array}$ & $\begin{array}{l}\text { Relative signal } \\
\quad \text { after } 4.5 \mathrm{~h} \\
\text { recovery }(\%)\end{array}$ & $\begin{array}{c}\text { Relative } \\
\text { signal after } \\
\text { additional } \\
6.8 \times 10^{12} \frac{\mathrm{GeV}}{\mathrm{cm}^{2}} \\
(2.1 \mathrm{Mrad})(\%)\end{array}$ \\
\hline $3 \mathrm{HF} 200 \mathrm{ppm} \mathrm{SC}$ & $26.3 \pm 2.1$ & $32.0 \pm 2.3$ & $14.6 \pm 1.1$ \\
\hline $3 \mathrm{HF} 500 \mathrm{ppm} \mathrm{SC}$ & $28.3 \pm 2.2$ & $38.7 \pm 2.7$ & $16.8 \pm 1.3$ \\
\hline $3 \mathrm{HF} 500 \mathrm{ppm}$ RHC & $78.8 \pm 5.6$ & $89.2 \pm 6.3$ & $75.9 \pm 5.4$ \\
\hline $3 \mathrm{HF} 1000 \mathrm{ppm} \mathrm{SC}$ & $30.2 \pm 2.5$ & $47.5 \pm 3.4$ & $20.7 \pm 1.7$ \\
\hline
\end{tabular}

\begin{tabular}{|c|c|c|c|c|}
\hline \multicolumn{5}{|c|}{$\overline{\bar{T} \text { Table } 4}$} \\
\hline Fiber & $\begin{array}{c}\text { Relative } \\
\text { signal after } \\
11.2 \times 10^{12} \frac{\mathrm{GeV}}{\mathrm{cm}^{2}} \\
(3.5 \mathrm{Mrad})(\%)\end{array}$ & $\begin{array}{c}\text { Relative } \\
\text { signal } \\
\text { after } 14 h \\
\text { recovery }(\%)\end{array}$ & $\begin{array}{c}\text { Relative } \\
\text { signal after } \\
\text { additional } \\
1.4 \times 10^{12} \frac{\mathrm{GeV}}{\mathrm{cm}^{2}} \\
(0.6 \mathrm{Mrad})(\%)\end{array}$ & $\begin{array}{l}\text { Relative signal } \\
\quad \text { after } 45 h \\
\text { recovery }(\%)\end{array}$ \\
\hline SCSF38 SC & $20.3 \pm 1.2$ & $34.6 \pm 1.9$ & $23.7 \pm 1.8$ & $36.2 \pm 2.7$ \\
\hline SCSF $81 Y 7$ SC & $31.1 \pm 2.5$ & $62.8 \pm 5.8$ & $40.2 \pm 3.3$ & $62.9 \pm 7.3$ \\
\hline SCSF $81 Y 8$ SC & $38.2 \pm 2.7$ & $67.2 \pm 3.6$ & $51.4 \pm 4.9$ & $61.7 \pm 6.7$ \\
\hline SCSF $81 Y 9$ SC & $27.6 \pm 3.4$ & $59.5 \pm 7.0$ & $32.1 \pm 5.0$ & not meas. \\
\hline BCF99-02 & $26.3 \pm 4.3$ & $46.7 \pm 5.8$ & $34.1 \pm 4.8$ & $51.3 \pm 6.7$ \\
\hline
\end{tabular}

\begin{tabular}{|l|c|c|}
\hline \hline \multicolumn{3}{|c|}{ Table 5 } \\
\hline Fiber & $\begin{array}{c}\text { Signal after irradiation } \\
\text { Signal before irradiation } \\
(\%)\end{array}$ & $\begin{array}{c}\text { Added constant term } \\
(\%)\end{array}$ \\
\hline 3HF 200 ppm Stand. cladding & $53.5 \pm 2.7$ & $1.09 \pm 0.24$ \\
3HF 500 ppm Stand. cladding & $56.9 \pm 2.8$ & $0.86 \pm 0.24$ \\
3HF 500 ppm Rad. hard cladding & $78.0 \pm 3.9$ & $0.76 \pm 0.24$ \\
3HF 1000 ppm Stand. cladding & $64.3 \pm 3.2$ & $1.14 \pm 0.24$ \\
SCSF38 Stand. cladding & $42.9 \pm 2.1$ & $3.00 \pm 0.31$ \\
SCSF81 Y9 Stand. cladding & $39.8 \pm 2.0$ & $2.26 \pm 0.28$ \\
BCF99-02 & $62.0 \pm 3.10$ & $1.63 \pm 0.26$ \\
\hline \hline
\end{tabular}




\begin{tabular}{|l|c|c|c|}
\hline \hline \multicolumn{3}{|c|}{ Table 6} \\
\hline Fiber type & $\begin{array}{c}0 \mathrm{~cm} \\
\text { PMT readout }\end{array}$ & $\begin{array}{c}90 \mathrm{~cm} \\
\text { Normalization } \\
\text { Point }\end{array}$ & $\begin{array}{c}220 \mathrm{~cm} \\
\text { Mirrored } \\
\text { Edge }\end{array}$ \\
\hline 3HF 200 ppm PMMA cladding & 175 & 54 & 28 \\
3HF 500 ppm PMMA cladding & 132 & 85 & 62 \\
3HF 500 ppm Fluorinated cladding & 97 & 60 & 36 \\
3HF 1000 ppm PMMA cladding & 112 & 70 & 43 \\
SCSF38 PMMA cladding & 360 & 100 & 36 \\
\hline \hline
\end{tabular}




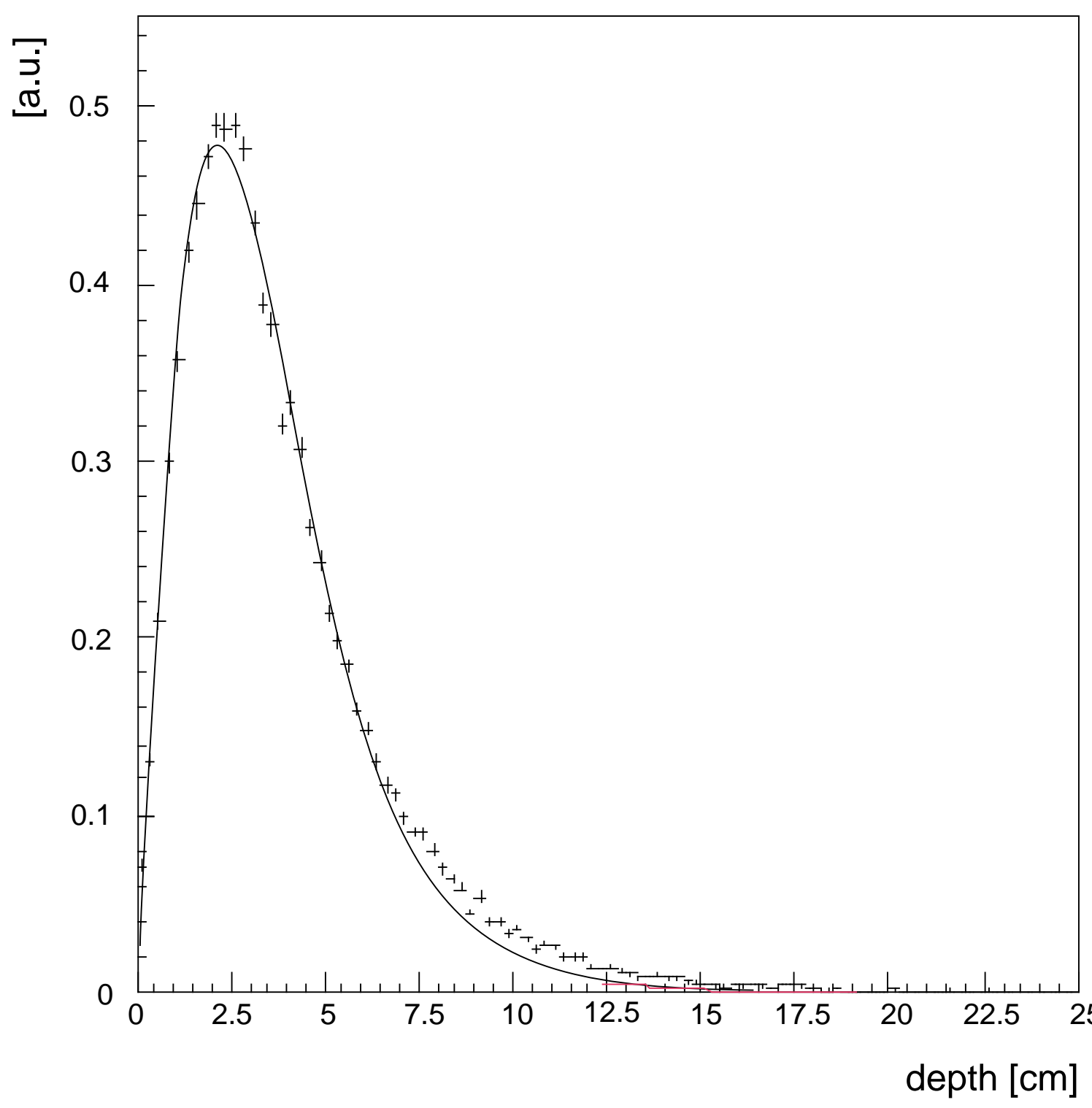

Figure 1 

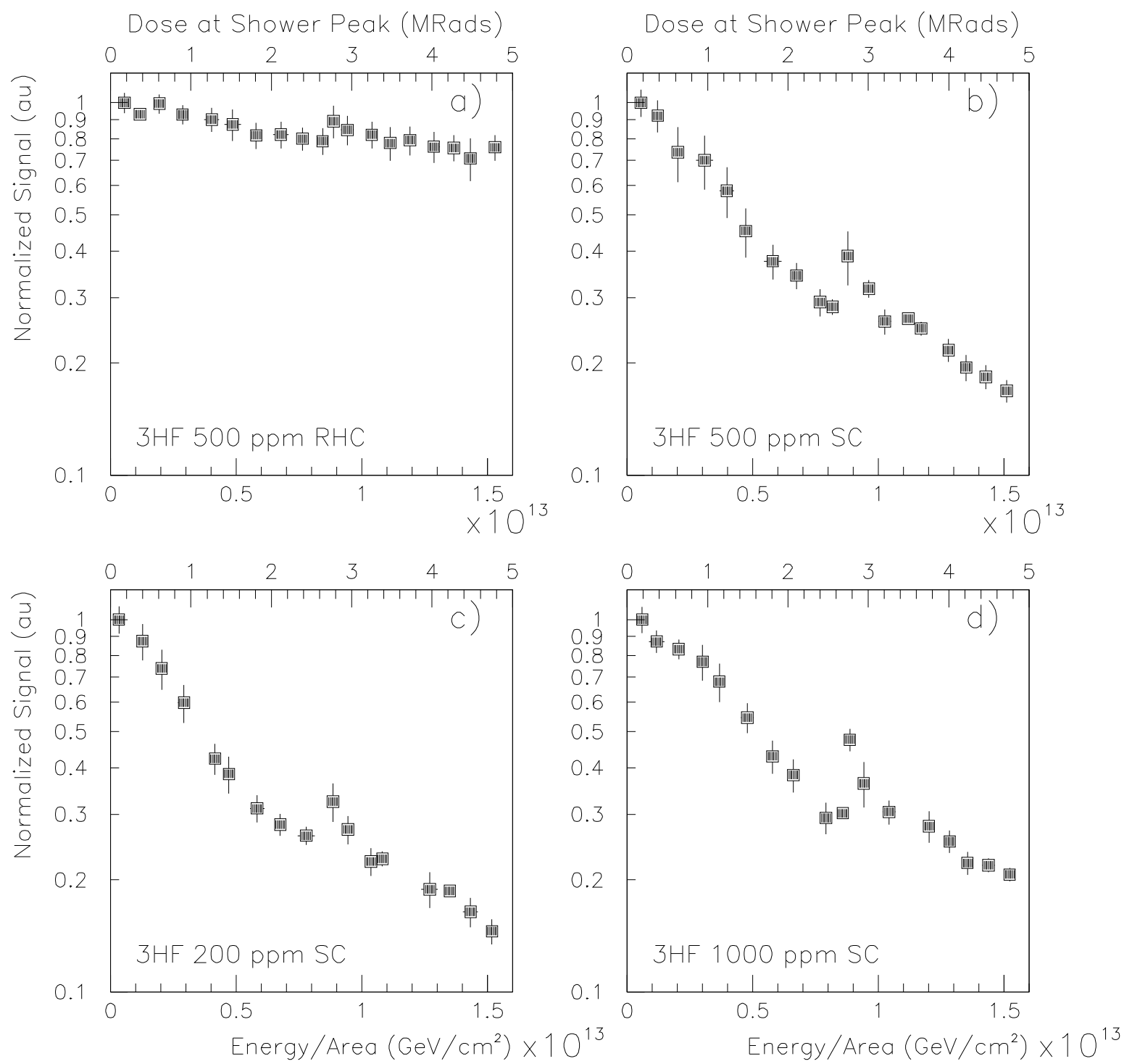

Figure 2 

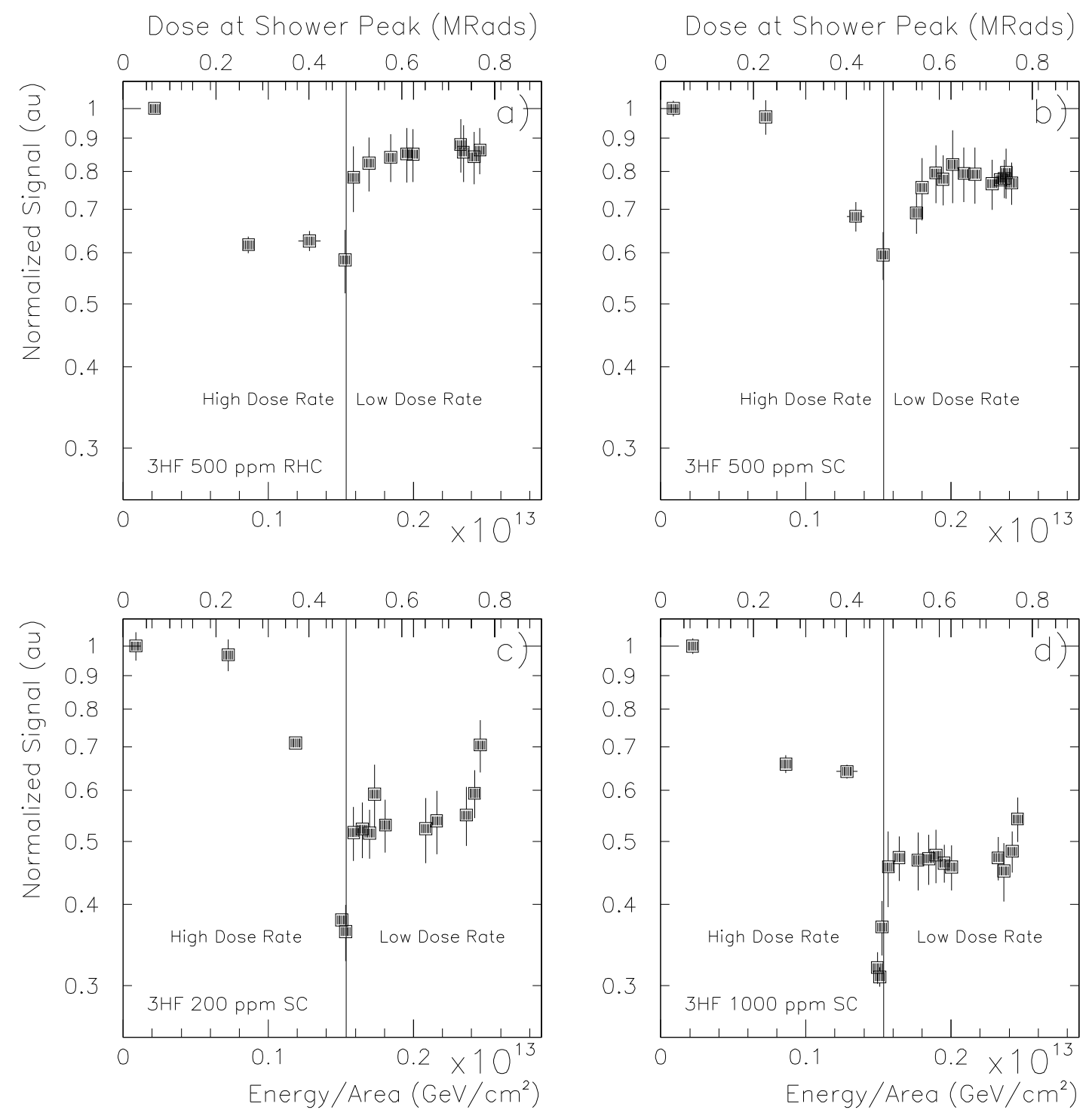

Figure 3 


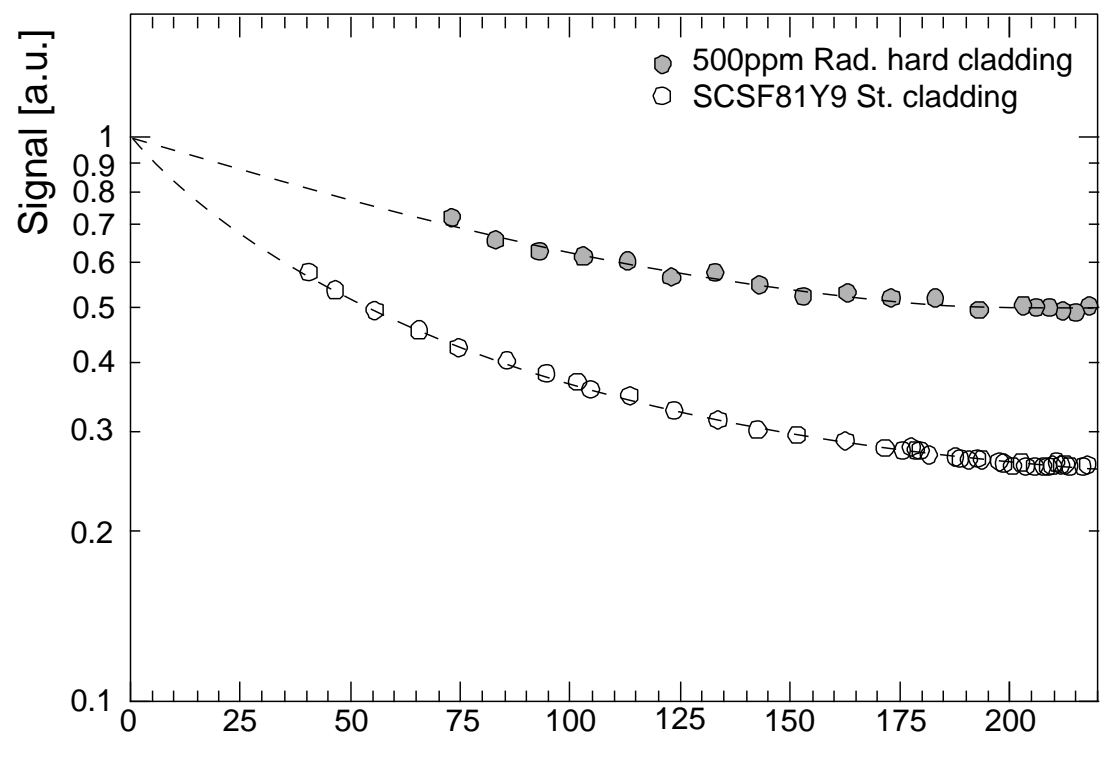

Distance from PM [cm]

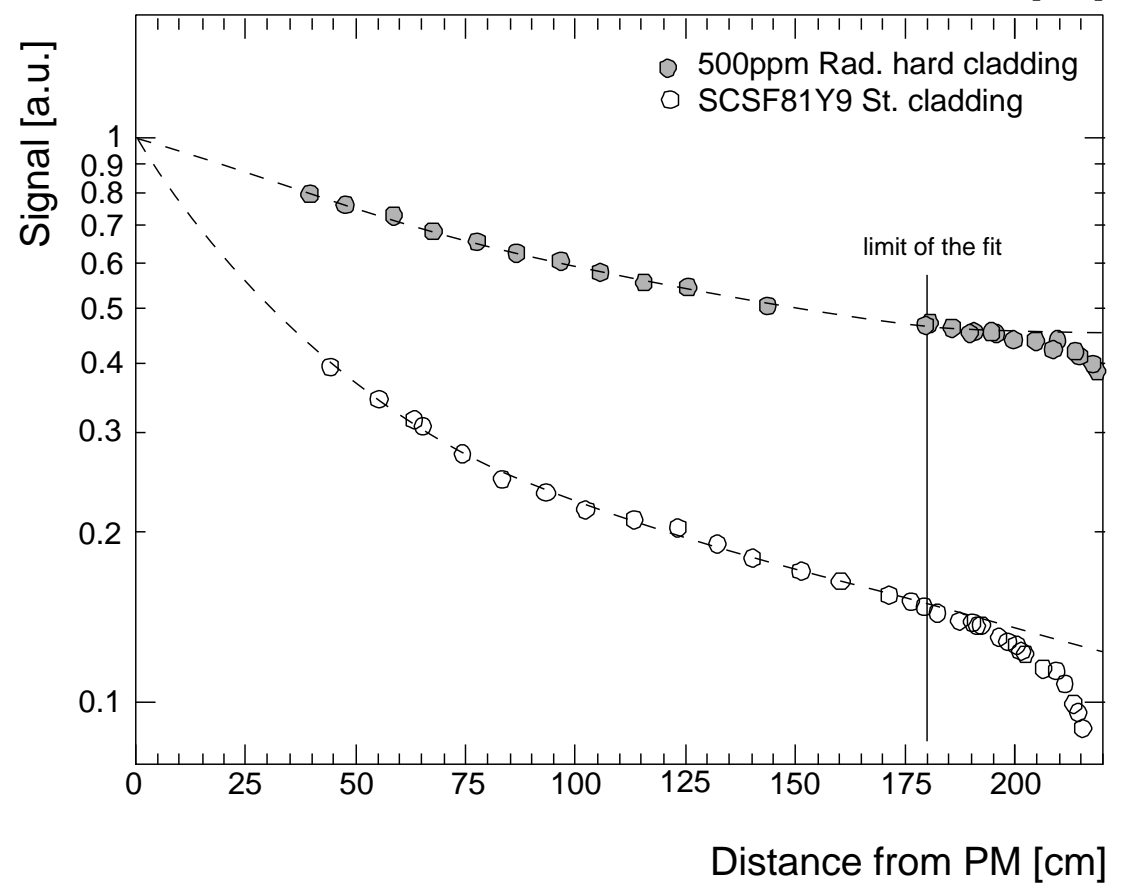

Figure 4 

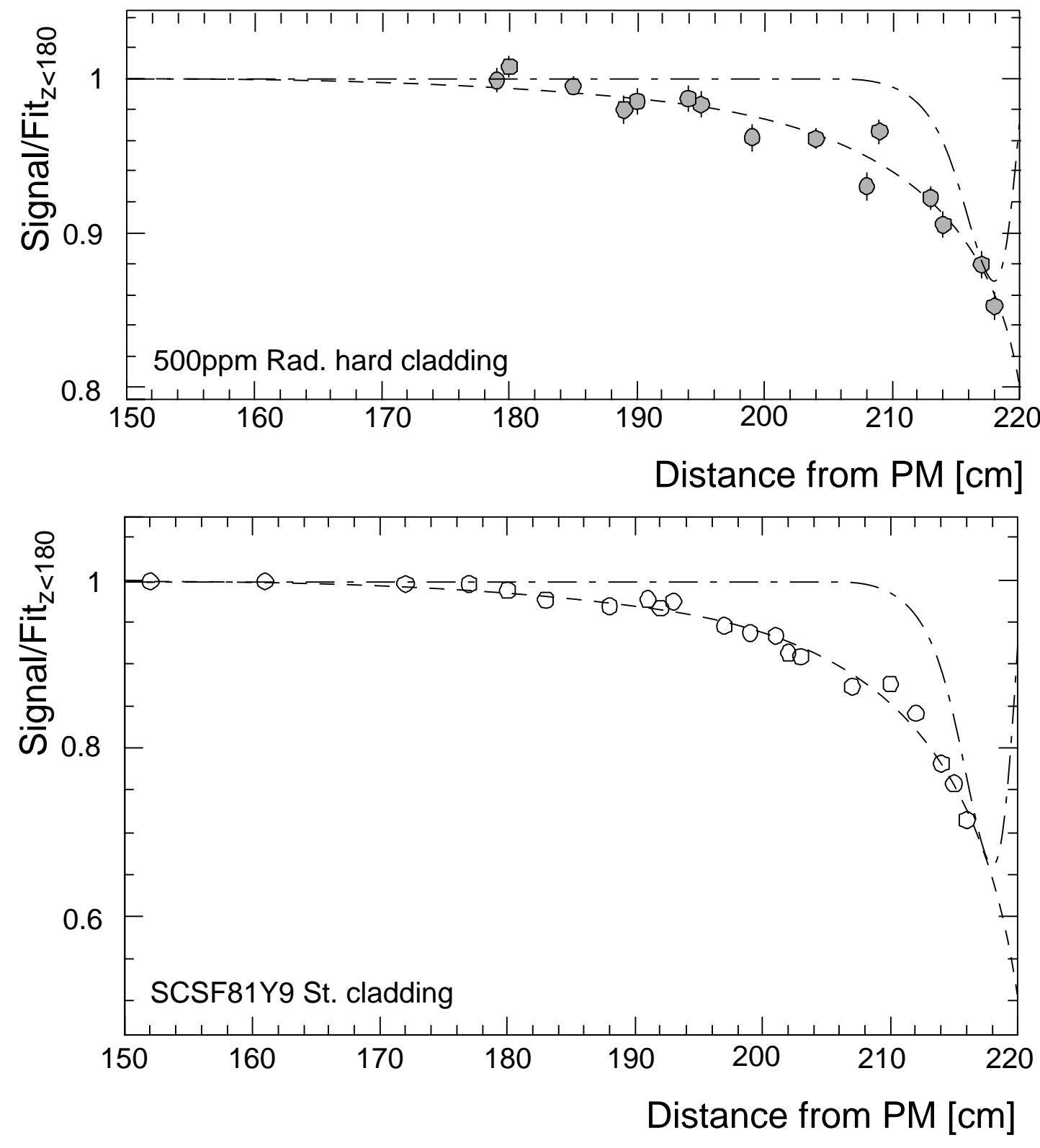

Figure 5 


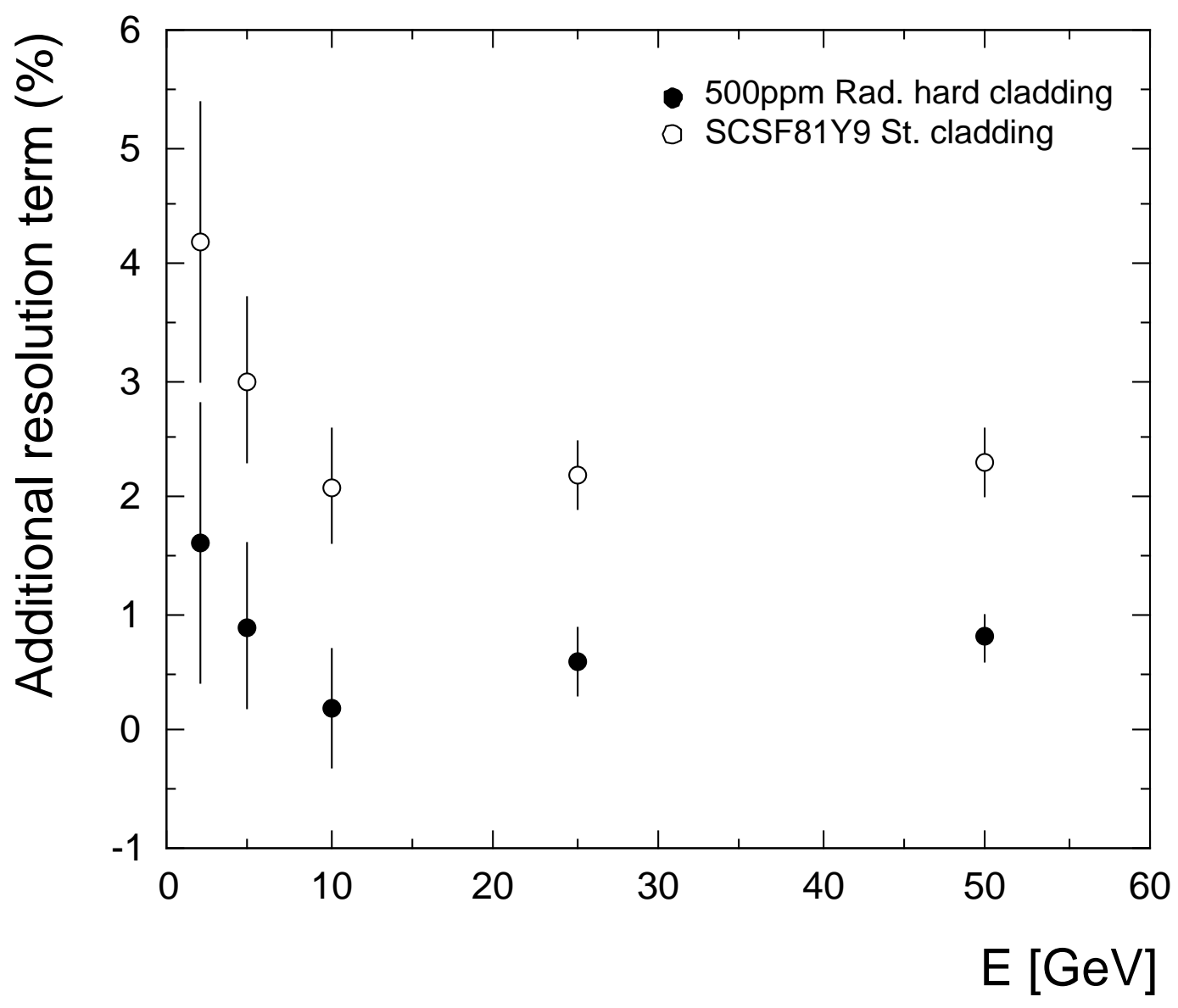

Figure 6 

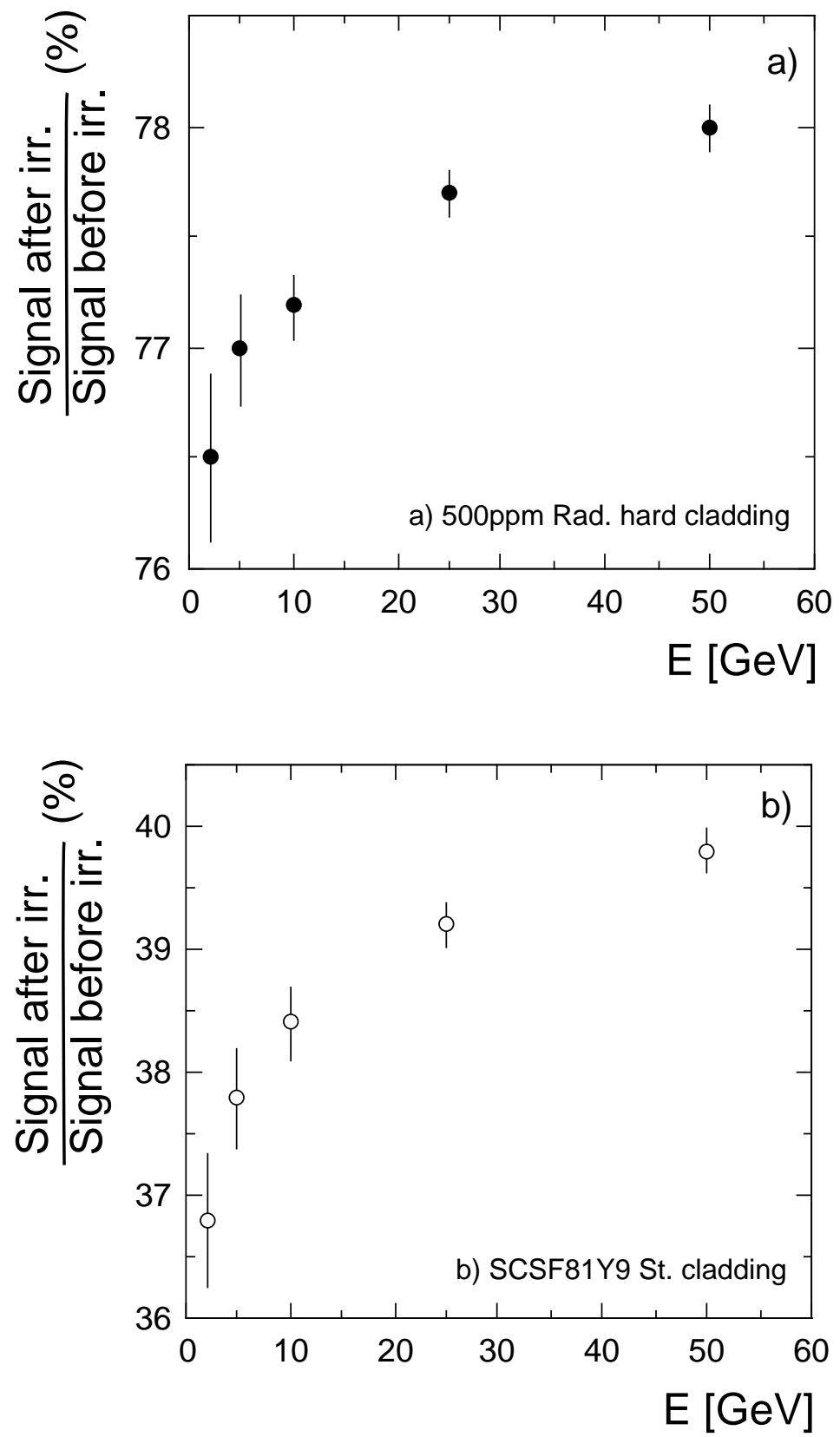

Figure 7 


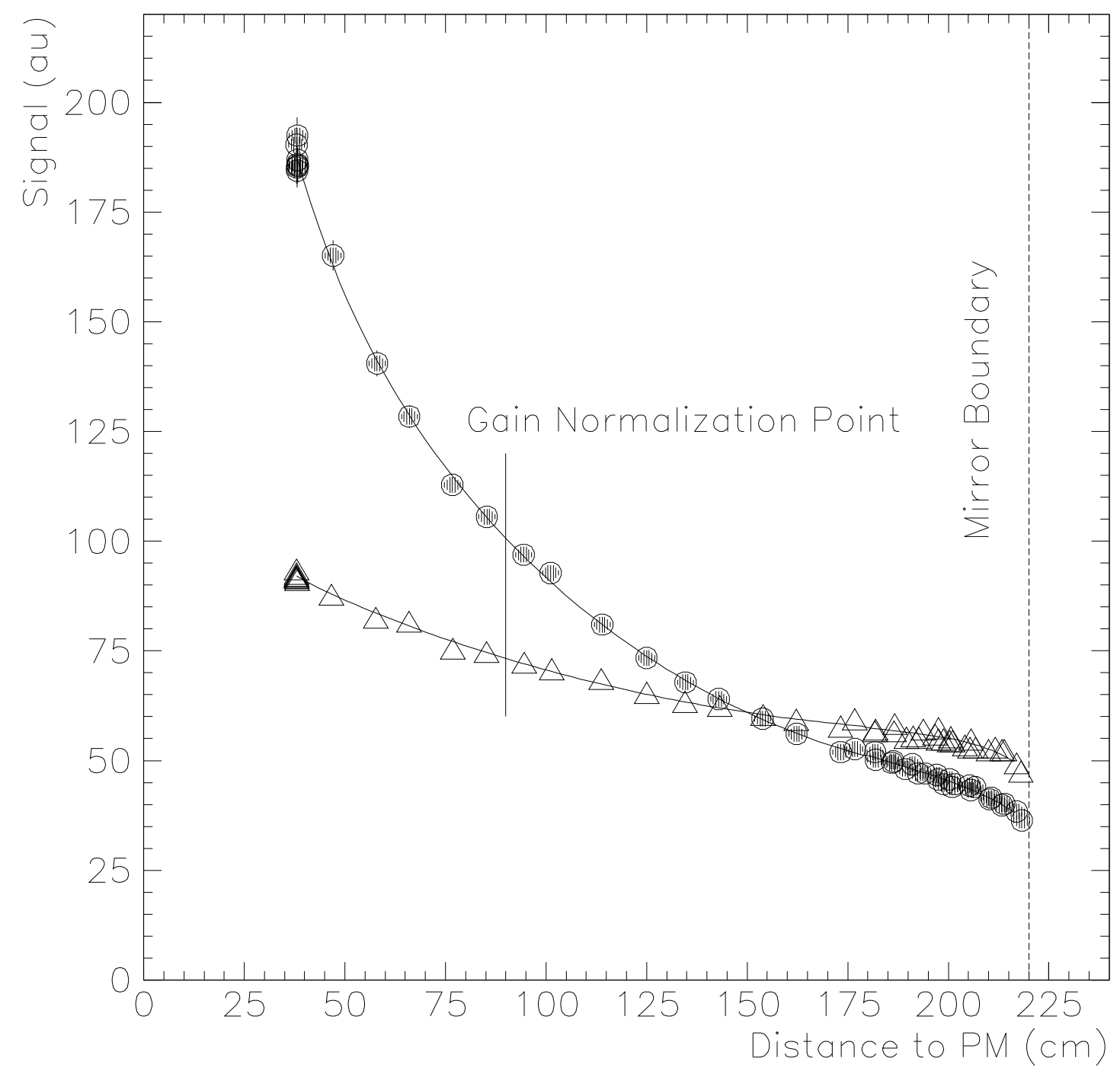

Figure 8 


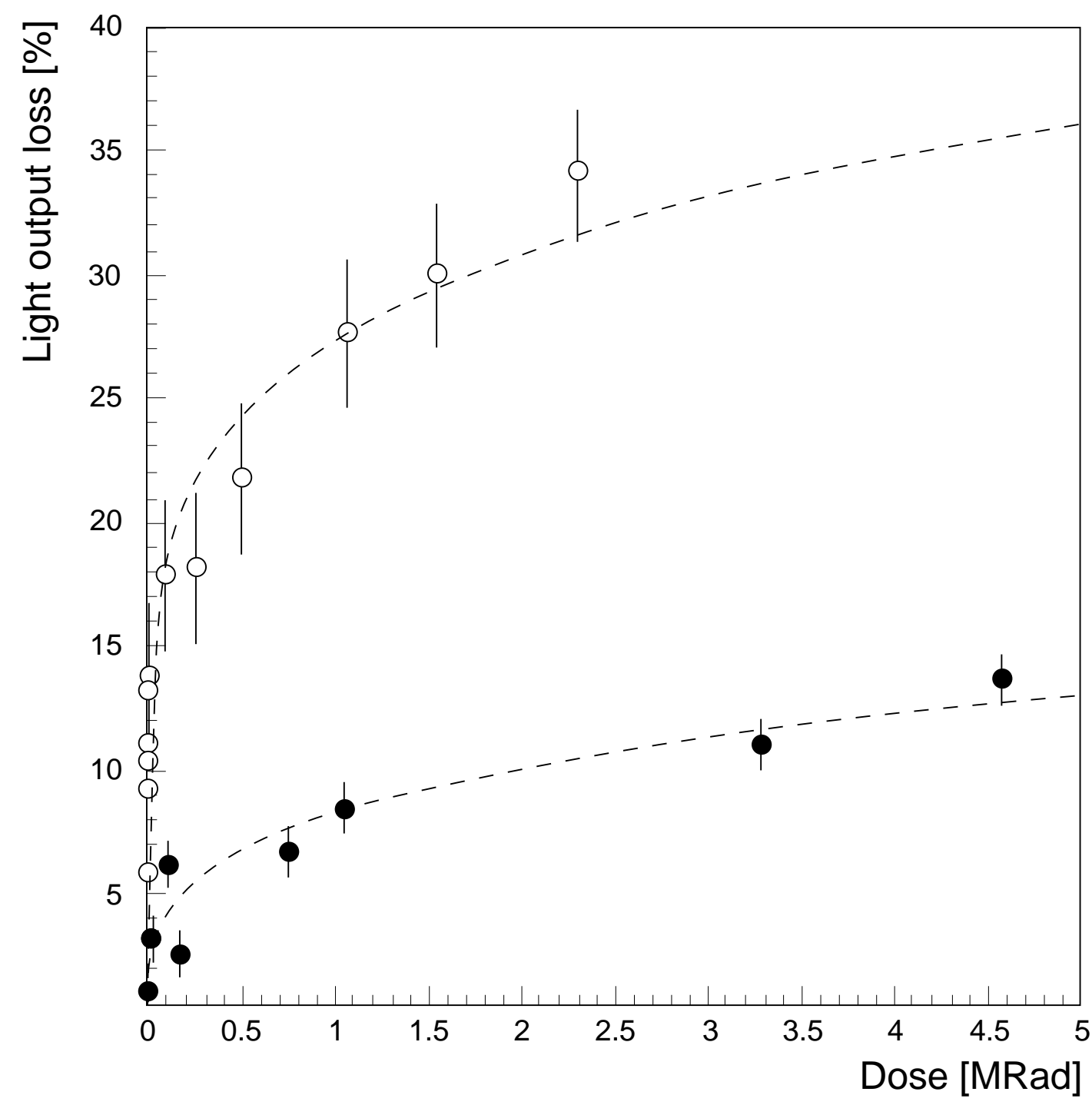

Figure 9 


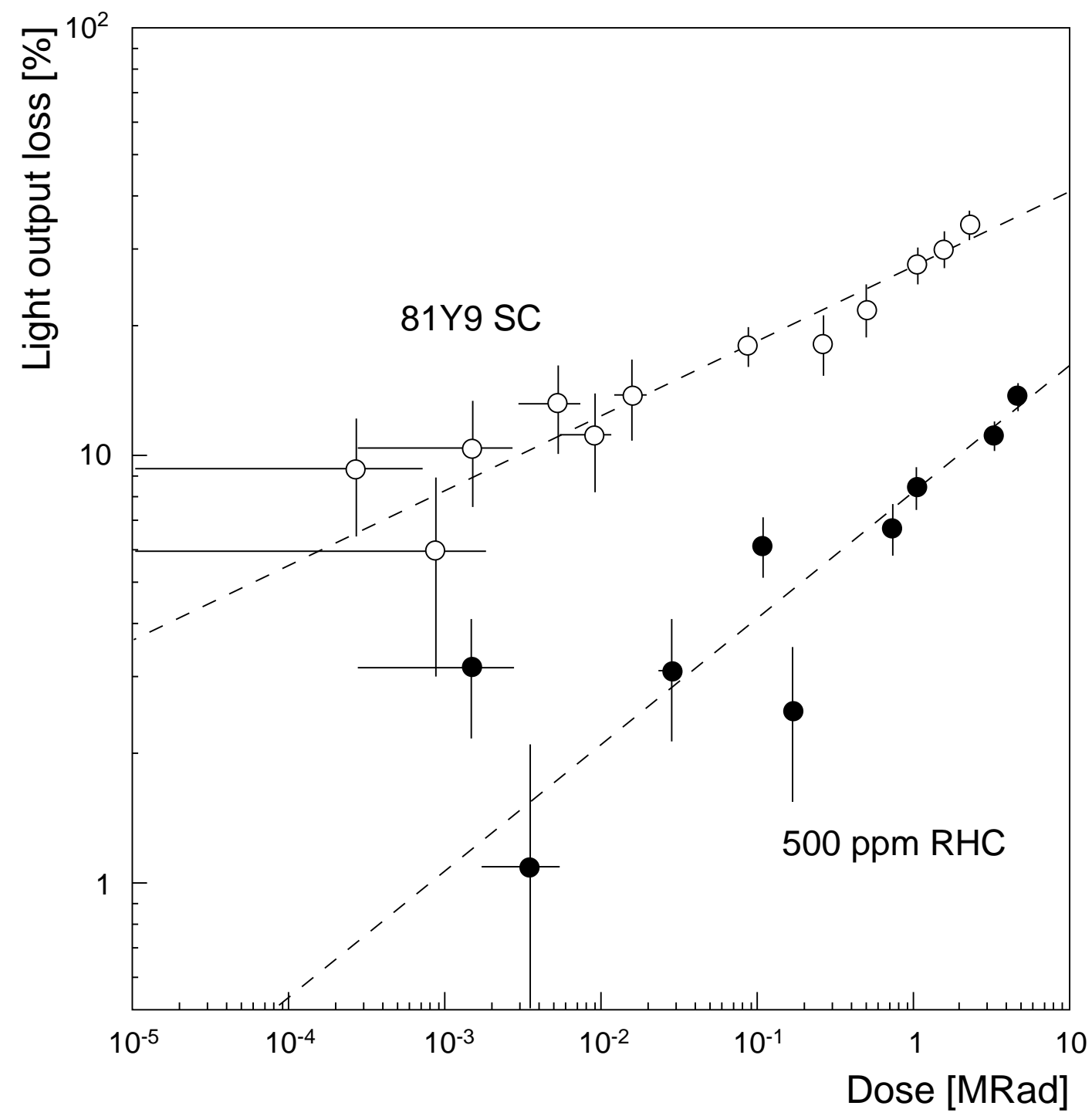

Figure 10 